Scientific Paper

\title{
Influence of gold marker for magnetic resonance imaging during prostate radiotherapy
}

\author{
Osamu TANAKA ${ }^{1, a}$, Hisao KOMEdA ${ }^{2}$, Shigeki HIROSE ${ }^{3}$, Takuya TANIGUCHI ${ }^{1}$, Kousei ONO ${ }^{1}$, Eiichi YAMA ${ }^{3}$, \\ Masayuki MATSUO ${ }^{4}$ \\ ${ }^{1}$ Department of Radiation Oncology, Murakimi Memorial Hospital, Gifu, Japan \\ ${ }^{2}$ Department of Urology, Gifu Municipal Hospital, Gifu, Japan \\ ${ }^{3}$ Division of Radiation Service, Gifu Municipal Hospital, Gifu, Japan \\ ${ }^{4}$ Department of Radiology, Gifu University School of Medicine, Gifu, Japan \\ ${ }^{a}$ E-mail address: c.bluered@gmail.com
}

(received 13 October 2017; revised 14 February 2018; accepted 20 June 2018)

\begin{abstract}
Gold markers (GM) are increasingly used for CT and MRI registration during in intensity-modulated radiation therapy for prostate cancer. Additionally, diffusion-weighted imaging has been adopted to measure the effects of radiotherapy for prostate cancer, similar to tests of blood prostate-specific antigen levels. As diffusion-weighted imaging (DWI) is often affected by a magnetic component, we evaluated the influence of GM on the Diffusion weighted images in three cases. We found that the size range of signal void of GM in MRI was 2-5 mm. We conclude that a GM would not affect the quality of DWI in daily practice.
\end{abstract}

Key words: prostate cancer; radiotherapy; MRI; fiducial marker; diffusion-weighted.

\section{Introduction}

Stereotactic body radiotherapy (SBRT) and intensitymodulated radiotherapy (IMRT) have led to great advances in radiotherapy (RT) for prostate cancer. However, RT requires precise radiation delivery, which has been increasingly facilitated by the use of gold marker (GM). Related research has reported that diffusion-weighted image (DWI) data (i.e., apparent diffusion coefficient, ADC) obtained via MRI can be used to detect the effects of RT on prostate cancer in a manner and correlates with prostate-specific antigen (PSA) levels in this context [1-3]. However, the size or iron component of a marker placed in the prostate might affect imaging results. Although DWI is known to be easily affected by metallic components, to our best knowledge, no previous reports have described whether changes in these images occurred from before to after GM placement. To address this issue, we conducted an initial case study of the clinical impacts of three patterns.

\section{Materials and Methods}

We examined following three patterns:

1) A decrease in ADC level was not observed from the beginning.

2) $\mathrm{ADC}$ level increased in peripheral zone before radiotherapy. ADC level decreased after radiotherapy.

3) ADC level did not change from before to after radiotherapy.
For this study, we used a VISICOILTM (VIS) $(0.35 \mathrm{~mm}$ in diameter, $10 \mathrm{~mm}$ in length), which is the most commonly used marker in our country, and a Gold AnchorTM (GA) $(0.28 \mathrm{~mm}$ in diameter, $10 \mathrm{~mm}$ in length). The visibility of GA on MRI is outstanding because it contains iron. We conducted a controlled trial of MR imaging of the VIS and GA markers at our hospital, placing GA and VIS on opposite sides of the prostate, respectively.

\section{Image acquisition}

\section{Parameters for T2*-weighted imaging (WI)}

$\mathrm{T} 2 *$-WI was performed using the following parameters: fast spin-echo; repetition time (TR)/echo time (TE) in ms, 700/18; number of sample (signals) averaged (NSA), two; number of phase-encoding steps (PES), 205; number of frequencyencoding steps (FES), 256; and typical spatial resolution (TSR) frequency/phase, $0.63 / 0.78$.

\section{Parameters for DWI}

DWI was performed using the following parameters: gradientecho; TR/TE in ms, 2264/70; NSA, eight; number of PES, 103; number of FES, 128; and TSR frequency/phase, 2.58/3.21.

\section{Parameters for planning CT}

Planning CT was performed at a slice thickness of $1.25 \mathrm{~mm}$, field of view of $40 \mathrm{~cm} \times 40 \mathrm{~cm}$, and settings of $460 \mathrm{~mA}$ and $120 \mathrm{kV}$. 


\section{Parameters for cone-beam CT}

Cone-beam CT was performed at a slice thickness of $2.5 \mathrm{~mm}$, $384 \times 384$ pixels, $80 \mathrm{~mA}$, and $125 \mathrm{kV}$.

An Optima CT580 (GE Medical Systems, Milwaukee, WI, USA) and Intera 1.5 Nova (Philips Medical Systems, Eindhoven, Netherlands) were used for CT and MRI, respectively. One blinded radiation oncologist performed ADC measurements. We outlined a region of interest (ROI) on the images obtained before and after treatment for comparison.

\section{Pattern 1. A decrease in the ADC level was not seen from the beginning}

A patient in his seventh decade visited our hospital for treatment and a follow-up evaluation. He had a history of chronic hepatitis C. An examination revealed that his PSA level had increased to $9.3 \mathrm{ng} / \mathrm{ml}$, and we underwent needle biopsy with 16 cores but found no evidence of cancer. One year later, his PSA level had increased further to $13.2 \mathrm{ng} / \mathrm{ml}$ and we performed a repeat biopsy. Prostate cancer with a Gleason Score (GS) of 5+4 was detected in one of 16 cores. However, as cancer was not clearly depicted on MRI, we assigned a stage of T1cN0M0 and planned a total RT dose of 78 Gy in 39 fractions. We next performed DWI before placing the VIS, but skewness of the clear image was not observed either before or after placement (Figure 1). After placing GM with a diameter of $0.35 \mathrm{~mm}$ and length of $10 \mathrm{~mm}$ (VISICOIL), no distortion was observed on DWI of the lesion.

\section{Pattern 2. An ADC level increased at the RT initiation by hormonal therapy before RT}

A patient in his seventh decade presented with dysuria, and the urology department was consulted. The patient had an increasing PSA level of $20.2 \mathrm{ng} / \mathrm{ml}$. Prostate cancer was detected via biopsy in 3 of 16 cores, and the tumor was staged as T3bN0M0 because extracapsular extension was detected on MRI. The GS was $4+4$ and high risk, and hormonal therapy was initiated. MR images before hormone therapy are shown in Figure 2a and $\mathbf{b}$. After 1.5 years, we planned to administer RT after his PSA level failed to decrease to $\leq 4 \mathrm{ng} / \mathrm{ml}$. Imaging depicted the disappearance of the tumor lesion after treatment (compared with an image before treatment with luteinizing hormone-releasing hormone therapy). Radiotherapy planning $\mathrm{CT}$ and MRI were obtained (Figure 2cde). GA was depicted clearly on T2*-WI. Skewness of the image was not observed on DWI (Figure 2e). The prostate cancer exhibited extracapsular extension in the left peripheral zone of the prostate (Figure 2ab). Hormonal therapy reduced the tumor to a size that was not recognizable on MR images. We performed CT/MRI for IMRT planning (after placing the GA marker; Figure 2cde). T2*-WI clearly recognized the GA. DWI recognized the GA without the influence of neighboring imaging. A total radiotherapy dose of $78 \mathrm{~Gy}$ in 39 fractions was administered.
Figure 1 Comparison of CT and MR image between before marker placement and after.

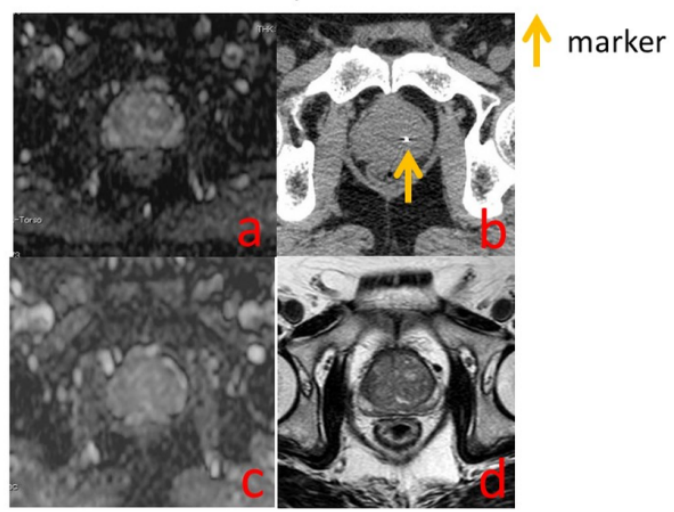

Figure 1. a) Diffusion-weighted imaging (DWI) before gold marker (GM) placement. b) Planning CT before GM placement. c) DWI after GM placement. d) T2-weighted imaging after GM placement

Figure 2 Comparison of CT and MR image between before marker placement and after.

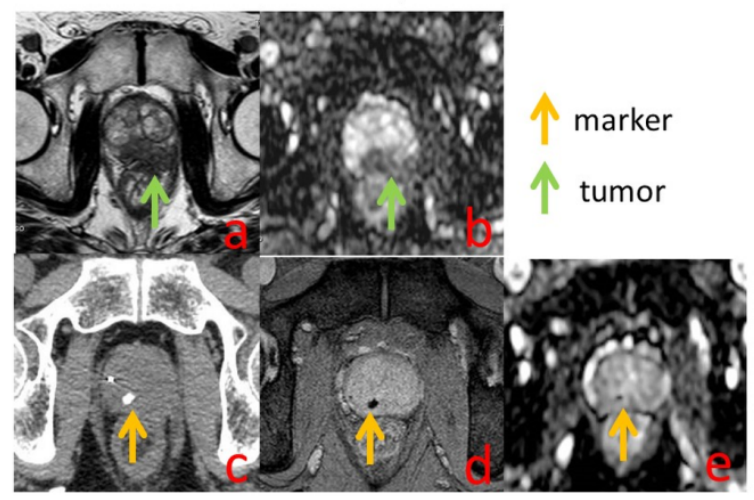

Figure 2. a) T2-weighted imaging (T2-WI) before gold marker (GM) placement. b) Diffusion-weighted imaging (DWI) before GM placement. c) Planning CT after GM placement. d) T2*-WI after GM placement. e) DWI after GM placement.

Figure 3 Comparison of CT and MR image between before marker placement and after.

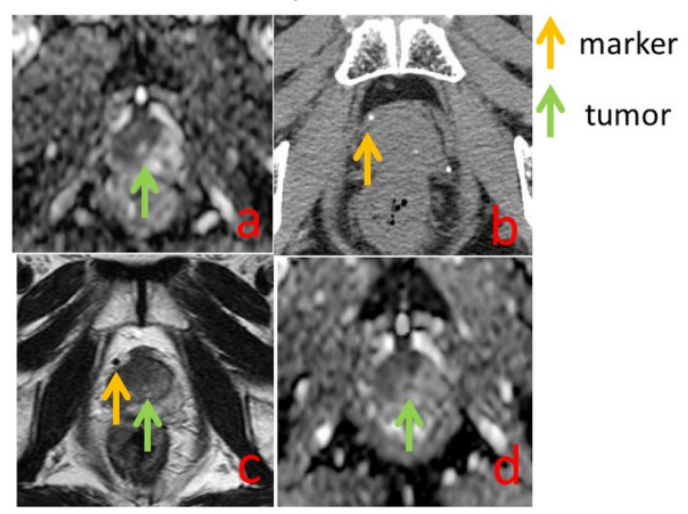

Figure 3. a) Diffusion-weighted imaging (DWI) before gold marker (GM) placement. b) Planning CT after GM placement. c) T2-weighted imaging (T2-WI) after GM placement. d) DWI after GM placement. 


\section{Pattern 3. ADC level did not change from before to after radiotherapy}

A patient in his seventh decade was referred from the hospital urology department with a high PSA level (13.2 ng/ml upon examination). Prostate cancer was detected by biopsy in 15 of 16 cores, and his GS was 3+4. In the right lobe, DWI revealed a decrease in the ADC level (mean $\mathrm{C}=0.758 * 10^{-3} \mathrm{~mm}^{2} / \mathrm{sec}$ ). We subsequently placed a VIS for IMRT and performed CT and MRI 3 weeks later, using conditions similar to those used before marker placement. The ADC level was $0.653 * 10^{-3}$ $\mathrm{mm}^{2} / \mathrm{sec}$, and no changes were observed on the image (Figure 3). The right transitional zone of the prostate appears as an area of low signal intensity (Figure 3a). A GM was placed on the capsule of the right frontal of the prostate (Figure 3bcd). No visual differences were observed from before to after GM placement (Figure 2ad). The total radiotherapy dose was 78 Gy in 39 fractions.

\section{Discussion}

The recent increase in patients with prostate cancer has allowed researchers to obtain precise information about various topics, including the effects of diagnostic and treatment methods and regimens. Current treatments for prostate cancer primarily include endocrine therapy, surgery and radiotherapy. PSA level is used as an indicator of recurrence after treatment, as well as of treatment outcomes. A previous report described the use of FDG-PET and MRI, which are considered diagnostic modalities, to reflect the effects of treatment [4-6]. Yamaguchi et al. reported that an increase in the ADC level at the prostate cancer significantly correlated with a decrease in the PSA at 6 months after radiotherapy [1].

Theoretically, we should be able to calculate the ADC level at a fixed-quantity level if we always do siege of ROI similarly. However, the image is often distorted by patient movement and metallic components, both of which can disrupt the uniformity of a magnetic field [7,8]. At our hospital, we provide an intramuscular injection of butylscopolamine 10 minutes before imaging to stop rectal movement during MRI.

MRI is very useful for depicting the outline of the prostate and the GM for CT and MRI registration for IMRT. However, to predict the effect of treatment using DWI is established in the absence of metal. Therefore, we performed imaging with two types of markers. Regardless of marker type, MRI (T2*WI) recognized approximately $2-5 \mathrm{~mm}$ as signal void. GA contains $0.5 \%$ of iron which was useful for MRI registration with CT. However, the effect of treatment cannot be detected via imaging if this susceptibility artifact affects the ADC level.

GA yielded a signal void of $5 \mathrm{~mm}$ on T2*-WI (Figure 2). Visually, there was no skewness on DWI, and no decrease in the real ADC level was observed. However, the signal void indicated that although the metal itself was indistinct on $\mathrm{T} 2{ }^{*}$ WI and DWI, the marker was slightly recognizable. However, the recognizable size was similar to that on $\mathrm{T} 2 *$-WI, and marker placement was not thought to clinically affect the ADC level. In other words, we observed a post-RT change in the ADC level based on an image of marker placement; when this finding was combined with the PSA level, it indicated the usefulness of DWI for evaluation.

We note that our study used the smallest-sized ironcontaining markers (Gold AnchorTM, $0.28 \mathrm{~mm}$; VISICOILTM, $0.35 \mathrm{~mm}$ ). However, larger markers (e.g., 0.75 $\mathrm{mm}$ ) or spherical markers (2 $\mathrm{mm}$ in diameter) may affect DWI. Our hospital is currently conducting the necessary studies to examine and compare GM of different sizes, shapes, and materials.

\section{Conclusion}

Marker placement did not affect the clinical use of MRI (DWI). However, this initial study used small markers $(0.28$ and $0.35 \mathrm{~mm})$. Studies involving larger markers and larger patient samples are needed.

\section{References}

[1] Yamaguchi H, Hori M, Suzuki O, et al. Clinical Significance of the Apparent Diffusion Coefficient Ratio in Prostate Cancer Treatment with Intensity-modulated Radiotherapy Anticancer Res. 2016;36(12):6551-6556.

[2] Qi WX, Zhang Q, Li P, et al. The predictive role of ADC values in prostate cancer patients treated with carbon-ion radiotherapy: initial clinical experience at Shanghai Proton and Heavy Ion Center (SPHIC). J Cancer Res Clin Oncol. 2016;142(6):1361-1367.

[3] Casares-Magaz O, van der Heide UA, Rørvik J, et al. A tumour control probability model for radiotherapy of prostate cancer using magnetic resonance imaging-based apparent diffusion coefficient maps. Radiother Oncol. 2016;119(1):111-116.

[4] Iannelli G, Caivano R, Rago L, et al. Diffusion-weighted magnetic resonance imaging in patients with prostate cancer treated with radiotherapy. Tumori. 2016;102(1):71-76.

[5] Decker G, Mürtz P, Gieseke J, et al. Intensity-modulated radiotherapy of the prostate: dynamic ADC monitoring by DWI at 3.0 T. Radiother Oncol. 2014;113(1):115-120.

[6] Morgan VA, Riches SF, Giles S, et al. Diffusion-weighted MRI for locally recurrent prostate cancer after external beam radiotherapy. AJR Am J Roentgenol. 2012;198(3):596-602.

[7] Park SY, Kim CK, Park BK, et al. Early changes in apparent diffusion coefficient from diffusion-weighted MR imaging during radiotherapy for prostate cancer. Int J Radiat Oncol Biol Phys. 2012;83(2):749-755.

[8] Song I, Kim CK, Park BK, Park W. Assessment of response to radiotherapy for prostate cancer: value of diffusion-weighted MRI at 3 T. AJR Am J Roentgenol. 2010;194(6):477-482. 